Trauma Berufskrankh $2008 \cdot 10$ [Suppl 2]:215-218

DOI 10.1007/s10039-007-1333-1

Online publiziert: 20. April 2008

(c) Springer Medizin Verlag 2008
H. Gollwitzer ${ }^{1,2}$

${ }^{1}$ Klinik für Orthopädie und Unfallchirurgie, Klinikum rechts der Isar, Technische Universität München, München

${ }^{2}$ Abteilung für Unfall- und Wiederherstellungschirurgie, BG-Unfallklinik Murnau, Murnau

\section{Extrakorporale Stoßwellentherapie}

\section{Gestörte Frakturheilung}

Die gestörte bzw. ausbleibende Knochenheilung stellt trotz Weiterentwicklung der konservativen Therapie sowie Verbesserung der Osteosyntheseverfahren eine bedeutende Komplikation in der Frakturbehandlung sowie nach elektiven Operationen wie Korrekturosteotomien oder Arthrodesen dar. Über eine ausbleibende Knochenheilung wurde nach Frakturbehandlung je nach Frakturtyp in $1->10 \%$ $[15,16,20]$ berichtet, bei elektiven Eingriffen beträgt die Pseudarthroserate etwa 1$5 \%[23,29]$. Die gestörte Knochenheilung kann meist auf eine oder mehrere der folgenden Ursachen zurückgeführt werden:

- lokale Durchblutungsstörung,

- Frakturinstabilität und/oder

- mangelnder Kontakt der Knochenenden (Knochendefekt) [30].

Abhängig von der Vitalität der Knochenfragmente und des Wachstumspotenzials des Knochens werden dementsprechend auch hypertrophe Pseudarthrosen mit deutlicher Kallusbildung von atrophen, areaktiven Pseudarthrosen unterschieden. Eine Sonderstellung nehmen septische Pseudarthrosen bei lokalen Infektionen ein.

\section{Extrakorporale Stoßwellentherapie}

\section{Grundlagen}

Zur Verbesserung der Knochenheilung wurde bereits eine Vielzahl an adjuvanten Therapieverfahren untersucht, u. a. die Ultraschalltherapie, die Elektrostimulation, die Magnetfeldbehandlung und die extrakorporale Stoßwellentherapie (ESWT). Die meisten dieser nichtinvasiven Verfahren, und insbesondere die ESWT, wurden deutlich häufiger zur Behandlung von Pseudarthrosen als primär adjuvant zur Frakturbehandlung eingesetzt. Entsprechend beziehen sich auch die Literaturangaben meist auf den Einsatz bei Pseudarthrosen $[2,12,25,26,27]$.

Bei der ESWT handelt es sich um ein physikalisches Verfahren, bei dem mechanische Einzelimpulse mit einem Druck- und Zuganteil (Stoßwelle) über eine externe Kopplung in das zu behandelnde Gewebe eingeleitet werden. Derartige Stoßwellen werden elektromagnetisch, piezoelektrisch oder elektrohydraulisch zunächst als divergierende Welle erzeugt und können dann über einen Reflektor vor dem Applikator fokussiert werden (fokussierte ESWT). An Grenzflächen mit unterschiedlicher Impedanz - wie zwischen Bindegewebe und Knochen - wird akustische Energie in mechanische, chemische und thermische Energie umgewandelt. Die daraus resultierende direkte mechanische Wirkung ist pro- portional zur Größe des Impedanzunterschieds [8].

Tierexperimentell liegt eine breite wissenschaftliche Grundlage zur Knochenstimulation durch ESWT vor, die osteogene Potenz konnte regelhaft bestätigt werden $[5,13,21,22]$. Als molekulare Wirkmechanismen werden v. a. eine Zellmembranhyperpolarisierung und Radikalbildung diskutiert, gefolgt von einer Stimulation von Knochenvorläuferzellen und der Expression von Wachstumsfaktoren [12].

Klinisch bestehen deutliche Hinweise auf eine Wirksamkeit der ESWT bei Pseudarthrosen. Die Heilungsraten wurden mit zwischen $41 \%$ und $88 \%$ angegeben, wobei ausschließlich Studien vom Evidenzgrad IV ohne Kontrollgruppe vorliegen $[2,12,25,26,27]$. Bisher fehlt ein echter Wirksamkeitsnachweis zur Stoßwellenbehandlung von Pseudarthrosen im Sinne einer randomisierten und kontrollierten Studie. Die Evidenz der ESWT in der Behandlung von Pseudarthrosen wurde bereits in einer früheren Publikation ausführlich diskutiert [12], weshalb der Fokus in der vorliegenden Arbeit auf die Applikation bei akuten Frakturen gerichtet wurde.

\section{Tierexperimentelle Untersuchungen zur Wirkung bei Frakturen und Osteotomien}

Der positive Effekt von kontrollierten Mikrobewegungen und Druckbelastungen auf den Frakturspalt wurde für die 


\begin{tabular}{|c|c|c|c|c|c|c|}
\hline Autor, Jahr & Tiermodell & Osteosynthese & Energie & Impulse & $\begin{array}{l}\text { Behandlungszeitpunkt } \\
\text { nach Fraktur/Operation }\end{array}$ & ESWT-Wirkung am Knochen \\
\hline $\begin{array}{l}\text { Seemann et al. } \\
1992[28]\end{array}$ & $\begin{array}{l}\text { Ratte (Osteotomie, } \\
\text { Knochendefekt) }\end{array}$ & Keine & $14 \mathrm{kV}$ & $\begin{array}{l}1-10 \times \\
80-240\end{array}$ & $\begin{array}{l}1,3,4,6,8,9,11,13 \\
15+17 \text { Tage }\end{array}$ & Keine Stimulation der Knochenheilung \\
\hline $\begin{array}{l}\text { Ekkernkamp } \\
\text { et al. } 1992 \text { [6] }\end{array}$ & $\begin{array}{l}\text { Schaf } \\
\text { (Osteotomie) }\end{array}$ & $\begin{array}{l}\text { V-förmiger } \\
\text { Fixateur externe }\end{array}$ & $\begin{array}{l}20 \mathrm{kV}, \\
24 \mathrm{kV}\end{array}$ & $1 \times 3000$ & 7 Tage & $\begin{array}{l}\text { Positiver Einfluss auf Knochenheilung } \\
\text { und Kallusproliferation }\end{array}$ \\
\hline $\begin{array}{l}\text { Haupt et al. } \\
1992[17]\end{array}$ & $\begin{array}{l}\text { Ratte } \\
\text { (Fraktur) }\end{array}$ & Keine & $\begin{array}{l}14 \mathrm{kV}, \\
18 \mathrm{kV}\end{array}$ & $5 \times 100$ & $2,5,9,14$ und 19 Tage & $\begin{array}{l}\text { Signifikante Beschleunigung } \\
\text { der Frakturheilung } \\
\text { Tendenziell Erhöhung der } \\
\text { Bruchstabilität }\end{array}$ \\
\hline $\begin{array}{l}\text { Augat et al. } \\
1995[1]\end{array}$ & $\begin{array}{l}\text { Schaf } \\
\text { (Osteotomie) }\end{array}$ & $\begin{array}{l}\text { Rigider } \\
\text { unilateraler } \\
\text { Fixateur externe }\end{array}$ & $\begin{array}{l}12 \mathrm{kV} \\
16 \mathrm{kV}\end{array}$ & $\begin{array}{l}2 \times 300- \\
3000\end{array}$ & $5+21$ Tage & $\begin{array}{l}\text { Kein signifikanter Unterschied der } \\
\text { Knochenheilung durch ESWT } \\
\text { Tendenziell Verminderung der mecha- } \\
\text { nischen Stabilität (dosisabhängig) }\end{array}$ \\
\hline $\begin{array}{l}\text { Uslu et al. } \\
1999 \text { [31] }\end{array}$ & $\begin{array}{l}\text { Kaninchen } \\
\text { (Knochendefekt) }\end{array}$ & Keine & $14 \mathrm{kV}$ & $3 \times 1000$ & 7, 14+21 Tage & $\begin{array}{l}\text { Vermehrte Kallusbildung } \\
\text { Neu gebildete Knochenstruktur } \\
\text { desorganisiert }\end{array}$ \\
\hline $\begin{array}{l}\text { Wang et al. } \\
2001[32]\end{array}$ & Hund (Fraktur) & 6-Loch-Platte & $14 \mathrm{kV}$ & $1 \times 2000$ & 0 Tage & $\begin{array}{l}\text { Verstärkte Kallusformation und höhere } \\
\text { Knochendichte nach } 12 \text { Wochen }\end{array}$ \\
\hline $\begin{array}{l}\text { Hsu et al. } \\
2003[18]\end{array}$ & $\begin{array}{l}\text { Kaninchen } \\
\text { (Osteotomie) }\end{array}$ & $\begin{array}{l}\text { Unilateraler } \\
\text { Fixateur externe }\end{array}$ & $\begin{array}{l}0,32 \\
\mathrm{~mJ} / \mathrm{mm}^{2}\end{array}$ & $2-3 \times 5000$ & 7, 21+35 Tage & $\begin{array}{l}\text { Beschleunigung der frühen } \\
\text { Frakturheilung mit Verbesserung der } \\
\text { mechanischen Eigenschaften }\end{array}$ \\
\hline $\begin{array}{l}\text { Wang et al. } \\
2003 \text { [33] }\end{array}$ & $\begin{array}{l}\text { Ratte } \\
\text { (Knochendefekt) }\end{array}$ & $\begin{array}{l}\text { 4-Loch-Platte mit } \\
\text { Zuggurtung }\end{array}$ & $\begin{array}{l}0,16 \\
\mathrm{~mJ} / \mathrm{mm}^{2}\end{array}$ & $1 \times 500$ & 6 Wochen & $\begin{array}{l}\text { Gesteigerte Expression der mRNA für } \\
\text { BMP-2, BMP-3, BMP-4 und BMP-7 }\end{array}$ \\
\hline $\begin{array}{l}\text { Chen et al. } \\
2004 \text { [3] }\end{array}$ & $\begin{array}{l}\text { Ratte } \\
\text { (Knochendefekt) }\end{array}$ & $\begin{array}{l}\text { 4-Loch-Platte mit } \\
\text { Zuggurtung }\end{array}$ & $\begin{array}{l}0,16 \\
\mathrm{~mJ} / \mathrm{mm}^{2}\end{array}$ & $1 \times 500$ & 12 Wochen & $\begin{array}{l}\text { Gesteigerte Rekrutierung und } \\
\text { Differenzierung mesenchymaler } \\
\text { Stammzellen } \\
\text { Expression von TGF- } \beta 1 \text { und VEGF-A }\end{array}$ \\
\hline $\begin{array}{l}\text { Chen et al. } \\
2004 \text { [2] }\end{array}$ & $\begin{array}{l}\text { Ratte } \\
\text { (Knochendefekt) }\end{array}$ & $\begin{array}{l}\text { 4-Loch-Platte mit } \\
\text { Zuggurtung }\end{array}$ & $\begin{array}{l}0,16 \\
\mathrm{~mJ} / \mathrm{mm}^{2}\end{array}$ & $1 \times 500$ & 8 Wochen & $\begin{array}{l}\text { Expression von Mediatoren des Kno- } \\
\text { chenwachstums (ERK, p-38-Kinase) } \\
\text { Steigerung der Aktivität von } \\
\text { alkalischer Phosphatase, und der } \\
\text { Synthese von Kollagen I, Kollagen II } \\
\text { und Osteokalzin sowie der } \\
\text { enchondralen Ossifikation }\end{array}$ \\
\hline $\begin{array}{l}\text { Wang et al. } \\
2004 \text { [35] }\end{array}$ & $\begin{array}{l}\text { Kaninchen } \\
\text { (Fraktur) }\end{array}$ & $\begin{array}{l}\text { Intramedullärer } \\
\text { Kirschner-Draht }\end{array}$ & $\begin{array}{l}0,18-0,47 \\
\mathrm{~mJ} / \mathrm{mm}^{2}\end{array}$ & $\begin{array}{l}1 \times 2000 \\
1 \times 4000\end{array}$ & 1 Woche & $\begin{array}{l}\text { Dosisabhängige Steigerung von } \\
\text { Knochenmasse, Kallusformation und } \\
\text { mechanischer Stabilität nach Fraktur } \\
\left(0,47 \mathrm{~mJ} / \mathrm{mm}^{2}\right)\end{array}$ \\
\hline
\end{tabular}

Knochenheilung bereits wiederholt nachgewiesen [7, 19]. Entsprechend wurden auch Osteosynthesematerialien hin $\mathrm{zu}$ weniger steifen Konstruktionen modifiziert, um eine begrenzte Mikrobewegung im Frakturspalt zu erlauben. Über einen ähnlichen Effekt aus kontrolliert eingebrachten Druck- und Zugkräften könnte auch die ESWT positiv zur Knochenheilung beitragen.

Die ESWT-bedingten tierexperimentell nachgewiesenen Effekte bei der Behandlung von Frakturen und Osteotomien sind in $\bullet$ Tab. 1 zusammengefasst. Während manche Autoren keine positive Beeinflussung der Knochenheilung und der Bruchstabilität durch ESWT beob- achten konnten $[1,28,31]$, berichtete die Mehrzahl der Untersucher über

- eine signifikante Beschleunigung der Frakturheilung [17, 18],

- vermehrte Kallusbildung [6, 31, 32, 35],

- gesteigerte Knochenmasse [35] und

- eine Verbesserung der mechanischen Eigenschaften des heilenden Knochens $[17,18,35]$.

Detaillierte Untersuchungen von Chen et al. [3, 4] und Wang et al. [33] ergaben eine signifikante Stimulation der Expression (knochenspezifischer) Wachstumsfaktoren wie BMP, TGF- $\beta 1$ und VEGF-A.
Die publizierten tierexperimentellen Untersuchungen sind aufgrund der Vielzahl möglicher Variablen der ESWT nur eingeschränkt vergleichbar. Die Unterschiede betreffen v. a. die Stoßwellenparameter wie Anzahl der Behandlungen, Impulszahl pro Behandlung, Behandlungsbeginn nach Fraktur/Osteotomie und Energieflussdichten. Zudem sind die applizierten Energien durch die Angabe verschiedener, nicht vergleichbarer physikalischer Größen wie Druck [bar], elektrische Spannung $[\mathrm{kV}]$ oder Energieflussdichte $\left[\mathrm{mJ} / \mathrm{mm}^{2}\right]$ inkomparabel. Der signifikante Einfluss von Behandlungsenergie und Impulszahl auf die Induktion der Osteoneogenese ist jedoch offensichtlich. 
Wie aus Tab. 1 ersichtlich, lässt sich aus den verschiedenen Studien noch keine klare Empfehlung zum Behandlungsprotokoll ableiten, eine positive Beeinflussung ist jedoch zu erwarten. Hierzu sind weitere, systematische Untersuchungen notwendig. $\mathrm{Zu}$ den weiteren biologischen Wirkungen der Stoßwellen wie der Induktion der Gefäßneubildung [34] und den antibakteriellen Effekten [10, 11] sei auf die weiterführende Literatur verwiesen.

Zusammenfassend konnten in den tierexperimentellen Untersuchungen in Abhängigkeit von den Behandlungsparametern meist eine signifikante Stimulation und Beschleunigung der Frakturheilung mit Verbesserung der biomechanischen Eigenschaften nachgewiesen werden. Ein optimales Behandlungsprotokoll muss noch über systematische Untersuchungsreihen etabliert werden.

\section{Klinische Ergebnisse bei Frakturen}

In hochwertigen randomisierten und kontrollierten Studien konnte eine Wirksamkeit der ESWT bei plantarem Fersensporn [14], Tendinosis calcarea [9] oder dem Tennisellenbogen [24] nachgewiesen werden. Auch für Pseudarthrosen liegt eine Großzahl qualitativ hochwertiger prospektiver Studien mit Konsolidierungsraten bis zu 90\% vor, ein Wirksamkeitsnachweis im Sinne einer kontrollierten Studie steht jedoch noch aus [12].

Während die beschriebenen Indikationen bereits breit in klinischen Studien untersucht wurden, gestaltet sich das Ergebnis einer Literaturrecherche in Medline und Pubmed zur Stoßwellentherapie bei Frakturen deutlich spärlicher. Bei der einzigen klinischen Untersuchung zur ESWT bei akuten Frakturen handelt es sich um eine prospektive, randomisierte Studie. Wang et al. [36] schlossen insgesamt 56 konsekutiv erfasste Patienten mit 59 Hochenergiefrakturen der langen Röhrenknochen der unteren Extremitäten ein. Die operative Stabilisierung erfolgte bei allen Patienten mit Nagel oder Platte, adjuvant wurde die Hälfte der Patienten noch während der Narkose mit 6000 Impulsen einer hochenergetischen Stoßwelle (o,62 $\mathrm{mJ} / \mathrm{mm}^{2}$ ) behandelt. Die Autoren berichteten über eine signifikante Reduktion der Rate an nicht konsolidierten Frakturen

Trauma Berufskrankh 2008 · 10[Suppl 2]:215-218 DOI 10.1007/s10039-007-1333-1

(c) Springer Medizin Verlag 2008

H. Gollwitzer

\section{Extrakorporale Stoßwellentherapie. Was ist die Evidenz?}

\section{Zusammenfassung}

Die extrakorporale Stoßwellentherapie (ESWT) ist zur Therapie von Knochen- und Weichteilpathologien etabliert. Typische Indikationen sind der Fersensporn, die Tendinosis calcarea und der Tennisellenbogen, aber auch Knochenpathologien wie die Pseudarthrose. Die vorliegende Arbeit fasst die in der Literatur verfügbaren Daten zur ESWT bei frischen Fakturen und Osteotomien zusammen. In den meisten Tierexperimenten wurden eine signifikante Beschleunigung der Heilung sowie eine Verbesserung der biomechanischen Eigenschaften durch ESWT nachgewiesen. Allerdings wurden dabei variable und nicht vergleichbare Behandlungsparameter eingesetzt, sodass ein optimales Behandlungsprotokoll noch etabliert werden muss. Zur klinischen Anwendung liegt eine

\section{Extracorporeal shock wave therapy. What is the evidence?}

\section{Abstract}

Extracorporeal shock wave therapy (ESWT) has been established as a unique treatment option for soft tissue and bone pathologies. Typical indications include plantar fasciitis, tendinosis calcarea, and tennis elbow, as well as bone pathologies such as nonunions. Regarding the efficacy of ESWT in acute fractures, the literature is herein reviewed. Significant and effective acceleration of fracture and osteotomy healing as well as improvement of biomechanical bone properties by ESWT has been demonstrated in most of the relevant published animal studies. Thus, variable and noncomparable treatment parameters have been applied, and an optimized treatment protocol still must be established. Clinical data are limited to one prospective prospektiv randomisierte Studie vor, die einen positiven Einfluss der noch am Operationstag durchgeführten ESWT auf die Heilung von Frakturen langer Röhrenknochen bestätigte. Allerdings müssen weitere klinische Studien zur Bestätigung der Wirksamkeit folgen. Während die Effektivität der ESWT bei Pseudarthrosen bereits in vielen Studien postuliert wurde und aufgrund der besten verfügbaren Evidenz empfohlen wird, kann zur ESWT bei frischen Frakturen aufgrund der begrenzten Datenlage derzeit noch keine Therapieempfehlung ausgesprochen werden.

\section{Schlüsselwörter}

Stoßwellentherapie - Lithotripsie · Fraktur . Osteotomie $\cdot$ Evidenzbasierte Medizin randomized trial, which demonstrated significant stimulation of healing of long bone fractures after ESWT was applied to the fracture site immediately after open reduction and internal fixation. Additional studies are required to corroborate the effectiveness of ESWT in acute fractures. Although the effectiveness of ESWT has been postulated in a myriad of trials investigating nonunions, published data are not sufficient to allow the recommendation of shock wave treatment for acute fractures also.

\section{Keywords}

Shock wave · Lithotripsy - Fracture .

Osteotomy · Evidence-based medicine 
nach 12 Wochen (Kontrolle 20\%, ESWT $11 \%)$ sowie eine signifikant beschleunigte Knochenheilung in der Gruppe mit Stoßwellenbehandlung. Als Limitationen dieser Studie müssen angeführt werden, dass insgesamt ein relativ kleines Patientenkollektiv untersucht und wesentliche Einflussfaktoren wie Raucher usw. nicht erfasst wurden. Somit ist die Untersuchung eher als Pilotstudie, denn als echter Wirksamkeitsnachweis zu werten.

\section{Fazit für die Praxis}

Für die ESWT zur Therapie von Frakturen existiert zwar eine prospektive randomisierte Studie mit Wirksamkeitsnachweis, die Literaturdaten sind jedoch auf diese eine Studie mit limitierter Aussagekraft beschränkt. Andererseits liegt für die ESWT bei Pseudarthrosen keine kontrollierte Studie vor, die Wirksamkeit wurde jedoch in einer Vielzahl hochwertiger prospektiver Studien untermauert. Ein Einsatz der ESWT bei Pseudarthrosen kann somit - insbesondere aufgrund der geringen Risiken und Nebenwirkungen und der günstigen Kosten-Nutzen-Relation - auf Basis der besten verfügbaren Evidenz empfohlen werden. Für die Beurteilung der Wirksamkeit extrakorporaler Stoßwellen bei akuten Frakturen reicht die momentane Datenlage nicht aus, hier müssen weitere kontrollierte Studien zur Klärung der korrekten Indikationsstellung und zur Bestätigung einer Wirksamkeit durchgeführt werden.

\section{Korrespondenzadresse}

\section{Dr. H. Gollwitzer}

Klinik für Orthopädie und Unfallchirurgie,

Klinikum rechts der Isar,

Technische Universität München, Ismaninger Straße 22, 81675 München gollwitzer@bone-and-joint.org

Interessenkonflikt. Der korrespondierende Autor gibt an, dass kein Interessenkonflikt besteht.

\section{Literatur}

1. Augat $P, C$ laes $L$, Suger $G$ (1995) In vivo effect of shock-waves on the healing of fractured bone. Clin Biomech (Bristol, Avon) 10: 374-378

2. Brandner H, Späth K (2001) Extrakorporale Stoßwellentherapie bei Knochenheilungsstörungen. Trauma Berufskrankh [Suppl 2] 3: 253-261
3. Chen YJ, Kuo YR, Yang KD et al. (2004) Activation of extracellular signal-regulated kinase (ERK) and p38 kinase in shock wave-promoted bone formation of segmental defect in rats. Bone 34: 466-477

4. Chen YJ, Wurtz T, Wang CJ et al. (2004) Recruitment of mesenchymal stem cells and expression of TGFbeta 1 and VEGF in the early stage of shock wavepromoted bone regeneration of segmental defect in rats. J Orthop Res 22: 526-534

5. Delius M, Draenert K, Al Diek Y et al. (1995) Biological effects of shock waves: in vivo effect of high energy pulses on rabbit bone. Ultrasound Med Biol 21: 1219-1225

6. Ekkernkamp A, Bosse A, Haupt G et al. (1992) Der Einfluß der extrakorporalen Stoßwellen auf die standardisierte Tibiafraktur am Schaf. In: Ittel TH, Sieberth H-G (Hrsg) Aktuelle Aspekte der Osteologie. Springer, Berlin Heidelberg New York, S 207210

7. Gardner MJ, Van der Meulen MCH, Demetrakopoulos D et al. (2006) In vivo cyclic axial compression affects bone healing in the mouse tibia. J Orthop Res 24: 1679-1686

8. Gerdesmeyer L, Maier M, Haake M et al. (2002) Physikalisch-technische Grundlagen der extrakorporalen Stoßwellentherapie (ESWT). Orthopade 31: 610-617

9. Gerdesmeyer L, Wagenpfeil S, Haake M et al. (2003) Extracorporeal shock wave therapy for the treatment of chronic calcifying tendonitis of the rotator cuff: a randomized controlled trial. JAMA 290: 2573-2580

10. Gerdesmeyer L, Eiff C von, Horn C et al. (2005) Antibacterial effects of extracorporeal shock waves. Ultrasound Med Biol 31: 115-119

11. Gollwitzer H, Horn C, Eiff C von et al. (2004) Antibakterielle Wirkung hochenergetischer extrakorporaler Stoßwellen: eine in vitro Untersuchung. Z Orthop Ihre Grenzgeb 142: 462-466

12. Gollwitzer H, Gloeck T, Brandner H (2006) Die Extrakorporale Stoßwellentherapie zur Behandlung von Knochenheilungsstörungen: was ist evidenzbasiert? Eine Literaturübersicht und eigene Ergebnisse. Trauma Berufskrankh 8: 142-152

13. Gollwitzer H, Gloeck T, Roessner M et al. (2006) Radial extracorporeal shock wave therapy (rESWT) induces bone formation in vivo: results of an animal model in rabbits. 52nd Annual Meeting of the Orthopaedic Research Society, Chicago, March 1922,2006

14. Gollwitzer H, Diehl P, Korff A von et al. (2007) Extracorporeal shock wave therapy for chronic painful heel syndrome: a prospective, double blind, randomized trial assessing the efficacy of a new electromagnetic shock wave device. J Foot Ankle Surg 46: 348-357

15. Hammacher ER, Van Meeteren MC, Van der Werken C (1998) Improved results in treatment of femoral shaft fractures with the unreamed femoral nail? A multicenter experience. J Trauma 45: 517-521

16. Hardy DC, Descamps PY, Krallis P et al. (1998) Use of an intramedullary hip-screw compared with a compression hip-screw with a plate for intertrochanteric femoral fractures. A prospective, randomized study of one hundred patients. J Bone Joint Surg Am 80: 618-630

17. Haupt G, Haupt A, Ekkernkamp A et al. (1992) Influence of shock waves on fracture healing. Urology 39: 529-532

18. Hsu RWW, Tai CL, Chen CYC et al. (2003) Enhancing mechanical strength during early fracture healing via shockwave treatment: an animal study. Clin $\mathrm{Bi}$ omech 18: S33-39
19. Jagodzinski M, Krettek C (2007) Effect of mechanical stability on fracture healing - an update. Injury [Suppl 1] 38: S3-10

20. Larsen LB, Madsen JE, Hoiness PR et al. (2004) Should insertion of intramedullary nails for tibial fractures be with or without reaming? A prospective, randomized study with 3.8 years' follow-up. J Orthop Trauma 18: 144-149

21. Maier M, Milz S, Wirtz DC et al. (2002) Grundlagenforschung zur Applikation extrakorporaler Stoßwellen am Stütz- und Bewegungsapparat. Eine Standortbestimmung. Orthopade 31: 667-677

22. Maier M, Hausdorf J, Tischer T et al. (2004) Knochenneubildung durch extrakorporale Stoßwellen: Einfluss der Energieflussdichte. Orthopade 33: 1401-1410

23. Muckley T, Schutz T, Srivastava S et al. (2003) Die Technik der tibiotalaren Arthrodese mit Kompressionsmarknagel. Unfallchirurg 106: 732-740

24. Pettrone FA, McCall BR (2005) Extracorporeal shock wave therapy without local anesthesia for chronic lateral epicondylitis. J Bone Joint Surg Am 87: 1297-1304

25. Rompe JD, Rosendahl T, Schollner C et al. (2001) High-energy extracorporeal shock wave treatment of nonunions. Clin Orthop Relat Res 387: 102-111

26. Schaden W (2000) Extrakorporale Stoßwellentherapie (ESWT) bei Pseudarthrosen und verzögerter Frakturheilung. Trauma Berufskrankh [Suppl 3] 2: 333-339

27. Schaden W, Fischer A, Sailler A (2001) Extracorporeal shock wave therapy of nonunion or delayed osseous union. Clin Orthop Relat Res 387: 90-94

28. Seemann O, Rassweiler J, Chvapil M et al. (1992) Effect of low-dose shock wave energy on fracture healing: an experimental study. J Endourol 6: 219223

29. Selber P, Filho ER, Dallalana R et al. (2004) Supramalleolar derotation osteotomy of the tibia, with $T$ plate fixation. Technique and results in patients with neuromuscular disease. J Bone Joint Surg Br 86: $1170-1175$

30. Stürmer KM (1996) Pathophysiologie der gestörten Knochenheilung. Orthopade 25: 386-393

31. Uslu MM, Bozdogan O, Guney S et al. (1999) The effect of extracorporeal shock wave treatment (ESWT) on bone defects. An experimental study. Bull Hosp Jt Dis 58: 114-118

32. Wang CJ, Huang HY, Chen HH et al. (2001) Effect of shock wave therapy on acute fractures of the tibia: a study in a dog model. Clin Orthop Relat Res 387: $112-118$

33. Wang FS, Yang KD, Kuo YR et al. (2003) Temporal and spatial expression of bone morphogenetic proteins in extracorporeal shock wave-promoted healing of segmental defect. Bone 32: 387-396

34. Wang CJ, Wang FS, Yang KD et al. (2003) Shock wave therapy induces neovascularization at the tendon-bone junction. A study in rabbits. J Orthop Res 21: 984-989

35. Wang CJ, Yang KD, Wang FS et al. (2004) Shock wave treatment shows dose-dependent enhancement of bone mass and bone strength after fracture of the femur. Bone 34: 225-230

36. Wang CJ, Liu HC, Fu TH (2007) The effects of extracorporeal shockwave on acute high-energy long bone fractures of the lower extremity. Arch Orthop Trauma Surg 127: 137-142 